\title{
A CORRECTION OF "THE NILSTUFE OF THE DIRECT SUM OF RANK 1 TORSION FREE GROUPS"
}

By

S. FEIGELSTOCK (Ramat Gan)

Professor R. Beaumont has pointed out an error in the proof of [1, theorem 1] which in turn gave rise to an error in the proof of theorem 3 . He also provided the following counter-example to theorem 3: Let $G_{1}$ be a rank one torsion free group of type $(1, \infty, 1, \infty, \ldots)$ and let $G_{2}$ be a rank one torsion free group of type $(0,1,0,1, \ldots)$. $G_{1}$ and $G_{2}$ are nil groups [2, theorem 121.1], but it is easy to show that $N\left(G_{1} \oplus G_{2}\right)=\infty$.

In what follows a theorem concerning homogeneous groups will be proved, as a consequence of which a weakened version of theorem 3 will be obtained.

THEOREM. Let $G=G_{1} \oplus G_{2}, G_{i}$ a homogeneous group, $1 \leqq i \leqq 2$, with $T\left(G_{1}\right)>$ $>T\left(G_{2}\right), T\left(G_{2}\right)$ not idempotent, and $v\left(G_{1}\right)=n$. Then $v(G) \leqq 2 n+1$.

Proor. Let $g \in G_{1}, h \in G$. For every multiplication on $G, T(g h) \geqq T(g) \geqq T\left(G_{1}\right)>$ $>T\left(G_{2}\right)$. Let $g h=g_{1}+g_{2}, g_{i} \in G_{i}, 1 \leqq i \leqq 2$. If $g_{2} \neq 0$, then $T(g h)=T\left(g_{2}\right)=T\left(G_{2}\right)$. A contradiction. Hence:

A) $G_{1} \cdot G \subseteq G_{1}$ and similarly

B) $G \cdot G_{1} \subseteq G_{1}$

for every multiplication on $G$.

Let $g, h \in G_{2}$. For every multiplication on $G, T(g h) \geqq 2 T\left(G_{2}\right)>T\left(G_{2}\right)$ since $T\left(G_{2}\right)$ is not idempotent. As above, this inequality yields:

C) $G_{2} \cdot G_{2} \leqq G_{1}$

for every multiplication on $G$.

A), B), and C) imply

D) $G \cdot G \subseteq G_{1}$ for every associative multiplication on $G$.

For every multiplication on $G, G^{2 n+2} \subseteq\left(G_{1}\right)^{n+1}$ by D). $\left(G_{1}\right)^{n+1}=0$ by virtue of the fact that $v\left(G_{1}\right)=n$.

COROLlaRY. Let $G=G_{1} \oplus G_{2}, G_{i}$ a rank one torsion free group, $v\left(G_{i}\right)=1,1 \leqq i \leqq 2$. If $T\left(G_{1}\right)>T\left(G_{2}\right)$ then $v(G) \leqq 3$.

\section{References}

[1] S. Feigetsrock, The nilstufe of the direct sum of rank 1 torsion free groups, Acta Math. Acad. Sci. Hungar., 24 (1973), 269-272.

[2] L. Fuchs, Infinite Abelian Groups, Vol. 2, Academic Press (New York and London, 1973).

(Received March 20, 1974)

BAR-ILAN UNIVERSITY

RAMAT-GAN, ISRAEL 